Ocular Oncology

and Pathology
Ocul Oncol Pathol 2018;4:23-32

DOI: $10.1159 / 000477408$
Received: July 7, 2016

Accepted after revision: May 1, 2017

Published online: July 5, 2017

\title{
Retinoblastoma: A Sixteen-Year Review of the Presentation, Treatment, and Outcome from a Tertiary Care Institute in Northern India
}

\author{
Usha Singh $^{\mathrm{a}}$ Deeksha Katoch ${ }^{\mathrm{a}}$ Savleen Kaur ${ }^{\mathrm{a}}$ Mangat Ram Dogra ${ }^{\mathrm{a}}$ \\ Deepak Bansal $^{\text {b }}$ Rakesh Kapoor ${ }^{\mathrm{c}}$ \\ ${ }^{a}$ Advanced Eye Centre, Department of Ophthalmology, and Departments of ${ }^{b}$ Pediatrics and ${ }^{\mathrm{c}}$ Radiotherapy, \\ Postgraduate Institute of Medical Education and Research, Chandigarh, India
}

\section{Keywords}

Intraocular retinoblastoma - Extraocular retinoblastoma .

Enucleation - Chemotherapy

\begin{abstract}
Purpose: To study epidemiology, demographic profile, clinical characteristics, and outcome in pediatric patients with retinoblastoma. Methods: This was a retrospective review of retinoblastoma patients of a tertiary institute from January 1st 1998 to December 31st 2014. Results: The study included 467 patients (618 eyes) with a mean age of $34.7 \pm 24.6$ months (median $=30 ; 15$ days to 144 months). Retinoblastoma was bilateral in 151 (32.3\%) and there were $61.7 \%$ males. Intraocular disease was seen in 301 patients (451 eyes [72.9\%]) and extraocular in 166 patients (167 eyes; 27.0\%). Out of the 347 (74.3\%) who received treatment, primary treatment was chemoreduction in 228 (65.7\%) and enucleation in 117 (33.7\%), while $25.6 \%$ of patients refused treatment and 151 (43.5\%) defaulted therapy. Local recurrence was seen in 20 (4.3\%), metastasis in $2(0.4 \%)$, and deaths in 13 (2.8\%) (average follow-up $28.5 \pm 44.4$ months). Histopathological high risk features were significantly less in the eyes that received chemoreduction (5.0\%) versus primary enucleation $(20.8 \%)(p<0.0004)$, but there was no difference in the
\end{abstract}

\section{KARGER}

(C) 2017 S. Karger AG, Basel

E-Mail karger@karger.com

www.karger.com/oop rate of metastasis, recurrence, and death between the two. Conclusions: The majority of retinoblastoma patients in our study had advanced disease, and nearly a third had extraocular extension. There were a significant number of therapy refusals and dropouts. Chemoreduction led to a significant decrease in the histopathological risk factors without affecting the outcomes.

(c) 2017 S. Karger AG, Basel

\section{Introduction}

Retinoblastoma is the most common primary intraocular malignancy of childhood. Despite advances in diagnosis and management, retinoblastoma still remains a considerable challenge especially in the developing world. More than $50 \%$ of patients die from the disease, and those who seek treatment are already at an advanced stage [1]. Survival in cases of extraocular retinoblastoma is even poorer due to intricate and ill-defined protocols for treatment $[1,2]$. Studies from our country reveal a large population of children with advanced stage at presentation with a mortality as high as $24 \%$ [3].

Poor ocular and patient survival in developing countries can be attributed mainly to the delay in the diagnosis

Prof. Usha Singh

Advanced Eye Center

Postgraduate Institute of Medical Education and Research

Chandigarh (India)

E-Mail drushasingh@gmail.com 
Table 1. Disease definitions used in the study

\begin{tabular}{ll}
\hline Group A & Tumor $\leq 3 \mathrm{~mm}$ in size and $>1.5 \mathrm{~mm}$ from foveola \\
\hline Group B & Tumor $>3 \mathrm{~mm}$ in size; $\leq 3 \mathrm{~mm}$ from foveola; $\leq 1.5 \mathrm{~mm}$ from disc \\
\hline Group C & Focal seeds $(\leq 3 \mathrm{~mm}) /$ discreet $\mathrm{Rb}$ with/without subretinal fluid $\leq 1$ quadrant \\
\hline Group D & Diffuse seeds/Rb with/without subretinal fluid $\geq 1$ quadrant \\
\hline Group E & $\begin{array}{l}\text { Extensive } \mathrm{Rb}>50 \% \text { of globe/diffuse infiltrative/anterior segment involvement/ } \\
\text { orbital cellulitis/phthisis/opaque media }\end{array}$ \\
\hline Extraocular disease & $\begin{array}{l}\text { Any extension of the tumor beyond the sclera into the orbit and/or into the optic } \\
\text { nerve substantiated by clinical examination and MRI }\end{array}$ \\
\hline $\begin{array}{l}\text { Locoregional disease } \\
\text { Orbital presentation with or without lymph node involvement }\end{array}$ \\
\hline $\begin{array}{l}\text { Metastasis } \\
\text { Advanced disease }=\text { International Intraocular Retinoblastoma Classification (IIRC) group D and E as well as } \\
\text { all extraocular Rb. Rb, retinoblastoma; MRI, magnetic resonance imaging. }\end{array}$ \\
\hline
\end{tabular}

and referral to a proper treatment center $[2,4]$. Poor socioeconomic conditions and lack of education contribute significantly to the existing problem of partial/incomplete treatment [4].

The Indian Council of Medical Research (ICMR) has reported a rise in the incidence of retinoblastoma cases in several hospital-based cancer registries in India [5]. As a tertiary care hospital in northern India, we have a dedicated retinoblastoma clinic since 1996 with a multidisciplinary team consisting of ophthalmologists, pediatricians, oncologists, and histopathologists. The aim of the present study is to discuss the epidemiology, demographic profile, clinical characteristics, and outcome of the recommended therapy in patients with retinoblastoma presenting to our center.

\section{Material and Methods}

Retinoblastoma clinic files and pediatric hemato-oncology records of patients presenting to the retinoblastoma clinic of the Advanced Eye Centre, Department of Ophthalmology, Postgraduate Institute of Medical Education and Research, India, between January 1st 1998 and December 31st 2014 (16 years), were reviewed. The demographic details of the patients namely age at onset of symptoms, age at presentation, gender, and details of residence (rural/urban) were collected. Patients were classified into rural background based on the criteria by the planning commission of India (defined as a town with a maximum population of 15,000 ). Disease characteristics such as laterality of the disease, family history, and presenting complaints were noted along with clinical signs including leukocoria, strabismus, and/or proptosis. Staging and treatment details were noted at baseline and at subsequent follow-up visits by detailed color-coded retinal diagrams along with clinical parameters. Repeated attempts were made to contact and trace the patients lost to follow-up, by phone and/or by home visits.

Tumors were classified into intraocular and extraocular category based on clinical presentation, radiological evaluation, and histopathological features. In addition to ocular examination, general physical examination was performed in all cases. International Intraocular Retinoblastoma Classification (IIRC) [6] and International Retinoblastoma Staging System [7] were used for classification. Eyes with bilateral retinoblastoma were grouped according to the eye with the more advanced disease. We re-classified intraocular disease for those presenting before 2006, according to current IIRC classification by reviewing their old examination under anesthesia (EUA) charts and imaging findings. Disease definitions are detailed in Table 1.

For unilateral intraocular disease, group A-C, focal therapies included laser photocoagulation or cryotherapy. With the establishment of newer treatment recommendations for intraocular retinoblastoma [8], neoadjuvant combination chemotherapy (globe salvage chemotherapy/first-line chemoreduction) was given to all bilateral cases. Amongst all bilateral cases, following chemoreduction, those with advanced intraocular disease (group D and E) underwent enucleation of the worse eye and conservative treatment (radiotherapy, laser photocoagulation or cryotherapy) of the better eye. The better eye was enucleated only if it did not respond to the above treatment methods. For unilateral advanced intraocular disease, enucleation was offered as first line of treatment. In case the patient opted against enucleation, globe salvage was attempted by chemoreduction, and after evaluating the initial response to 2 or 3 cycles of chemotherapy, local therapy (laser photocoagulation or cryotherapy), secondary enucleation, and/or external beam radiotherapy was administered. Adjuvant chemotherapy for 6 cycles was given at 3 weekly intervals to those patients who had histopathological risk factors (HRF). Intravitreal chemotherapy in the form of melphalan was offered to patients after the year 2013 in addition to chemotherapy when they had intraocular retinoblastoma with vitreous seeds [9]. 
Extraocular disease was treated by 2-3 cycles of chemoreduction followed by secondary enucleation, external beam radiation and adjuvant chemotherapy for an additional 6-10 cycles (to complete 12 cycles of chemotherapy). Metastatic work included hematological investigations, bone marrow aspiration, cerebrospinal fluid analysis, computerized tomography and/or magnetic resonance imaging (MRI) of the brain before the year 2012 and MRI alone after the year 2012, ultrasound abdomen in patients who were clinically group $\mathrm{E}$ and onwards.

Two chemotherapy regimens were used: vincristine, cisplatin, etoposide, cyclophosphamide (OPEC) until March 2007, and vincristine, etoposide, carboplatin (VEC) thereafter. OPEC was administered over $55 \mathrm{~h}$, beginning with vincristine $\left(1.5 \mathrm{mg} / \mathrm{m}^{2}\right)$ and cyclophosphamide $\left(600 \mathrm{mg} / \mathrm{m}^{2}\right)$ followed by cisplatin $\left(80 \mathrm{mg} / \mathrm{m}^{2}\right)$ and etoposide $\left(200 \mathrm{mg} / \mathrm{m}^{2}\right)$. It was replaced by VEC regime, which is the current practice. VEC comprises of vincristine $\left(1.5 \mathrm{mg} / \mathrm{m}^{2}\right)$, etoposide $\left(600 \mathrm{mg} / \mathrm{m}^{2}\right)$, and carboplatin $\left(300 \mathrm{mg} / \mathrm{m}^{2}\right)$, all administered on the same day. Histopathology (HP) reports of enucleated eyes were analyzed for HRF predictive of metastasis namely involvement of optic nerve (retrolaminar portion with positive resection limit), choroidal invasion, anterior segment involvement, and scleral and extrascleral involvement. Choroidal invasion was classified as non-massive if $<3 \mathrm{~mm}$ and massive if $\geq 3 \mathrm{~mm}$ in any dimension.

Patients were followed up periodically. Serial monitoring of the tumors was done with ophthalmic ultrasound and examination under anesthesia. At each visit, the size and extent of lesion, regression, or progression was recorded. After completion of therapy, follow-up examination was done every 3 months for the first 2 years to rule out any local recurrence and/or side effects of therapy followed by 4- to 6-monthly examination depending on the initial stage of disease. Recurrence was defined as progression in tumor size/reappearance or new lesion 3 months after completion of primary therapy plus adjuvant therapy. Treatment defaulter was defined as a patient who abandoned treatment midway (dropout). The outcome was defined in terms of globe salvage rate, recurrence, and death.

\section{Statistical Analysis}

Statistical analysis was done using the Statistical Package for Social Sciences (SPSS; IBM SPSS Statistics for windows, version 21.0; Armonk, NY, USA). Values were expressed as means \pm standard deviation (SD) along with percentages expressed as proportions. Categorical variables were compared using the $\chi^{2}$ or Fisher's exact test. In cases of bilateral disease, the eye with the higher group was included to analyze the severity of disease. A $p$ value $<0.05$ was considered statistically significant.

\section{Results}

Four hundred and sixty-seven patients (618 eyes) with retinoblastoma presented during the study period. Our data of 467 patients correspond to an average of 29 new cases/year. Retinoblastoma was bilateral in 151 patients $(32.3 \%)$ and unilateral in $316(67.6 \%)$. Two hundred and fifty (53.5\%) patients belonged to rural area, while 217
Table 2. Distribution of clinical features on presentation in the study cohort

\begin{tabular}{|c|c|}
\hline Chief symptom & $n(\%)$ \\
\hline Leukocoria & $375(60.6)$ \\
\hline Strabismus & $105(16.9)$ \\
\hline Hyperemia of eye & $50(8.1)$ \\
\hline Neovascularization & $48(7.8)$ \\
\hline Proptosis & $27(4.4)$ \\
\hline Glaucoma & $3(0.49)$ \\
\hline (7) Others & $10(1.6)$ \\
\hline
\end{tabular}

(46.5\%) came from urban areas. A total of 74 patients (12.9\%) were "below poverty line" (below poverty line is an economic benchmark and poverty threshold defined by the World Bank as income less than USD 1.25 per day; and in India income less than USD 0.43 in rural areas and about USD 0.53 in urban areas by the economic criteria). Two hundred and eighty-eight boys (61.7\%) and 179 girls $(38.3 \%)$ were affected. Nineteen children $(4.0 \%)$ had at least one other relative who had been diagnosed with retinoblastoma. Of these, 3 (15.8\%) had unilateral disease, while $16(84.2 \%)$ were bilateral. One patient screened for retinopathy of prematurity had bilateral retinoblastoma diagnosed at 1 month of age. None of the patients had history of any other systemic cancer in the family as per records.

\section{Age at Presentation}

Mean age at presentation was $34.7 \pm 24.6$ months (median $=30$ months; range 15 days to 144 months). The mean age at presentation in unilateral disease was $36.5 \pm$ 24.0 months (median $=13$; range $2-144$ months) and 30.9 \pm 25.4 months (median $=3$; range 15 days to 120 months) in the bilateral group of children. The mean age in the bilateral group was lower than in the unilateral group, but this difference was not statistically significant $(p=0.342)$. Delay in presentation from the onset of symptoms/signs was computed for all patients, and was found to be $8.3 \pm$ 13.8 months (range 0-84 months; median $=3$ months). Four hundred and sixteen patients $(89.0 \%)$ were diagnosed in their first 5 years, while $51(10.9 \%)$ presented after 5 years of age. Of the late presenters, the majority were unilateral $(74.5 \%)$. There was no difference in the gender distribution of the late presenters $(p=0.065)$. Four bilateral cases unusually presented between 9 and 10 years of age. The age at presentation of those who were below poverty line $(26.4 \pm 21.2$ months) was comparable to those who were above $(22.7 \pm 23.3$ months $)(p=0.33)$.

Ocul Oncol Pathol 2018;4:23-32 DOI: $10.1159 / 000477408$ 
Table 3. Analysis of unilateral versus bilateral disease at presentation

\begin{tabular}{|c|c|c|c|c|c|c|c|c|c|c|}
\hline & \multirow[t]{2}{*}{ Eyes, $n^{\mathrm{a}}$} & \multirow{2}{*}{$\begin{array}{l}\text { Globe } \\
\text { salvage } \\
\text { rate, \% }\end{array}$} & \multicolumn{4}{|c|}{ Unilateral disease $(n=316)$} & \multicolumn{4}{|c|}{ Bilateral disease $(n=151 \times 2)(302$ eyes $)$} \\
\hline & & & eyes, $n$ & $\begin{array}{l}\text { recur- } \\
\text { rences, } n\end{array}$ & deaths, $n^{\mathrm{a}}$ & $\begin{array}{l}\text { metastasis, } \\
n^{\mathrm{a}, \mathrm{b}}\end{array}$ & eyes, $n$ & $\begin{array}{l}\text { recur- } \\
\text { rences, } n\end{array}$ & deaths, $n^{\mathrm{a}}$ & metastasis, $n^{\mathrm{a}, \mathrm{b}}$ \\
\hline Group A & 22 & 100 & - & 0 & - & - & 22 & 0 & - & - \\
\hline Group B & 58 & 100 & 2 & 0 & - & - & 56 & 0 & - & - \\
\hline Group C & 20 & $94.7^{\mathrm{b}}$ & - & 0 & - & - & 20 & 0 & - & - \\
\hline Group D & 47 & 17.1 & 21 & 0 & 0 & 0 & 26 & 0 & 0 & 0 \\
\hline Group E & 304 & 0 & 128 & 6 & 1 & 0 & 176 & 7 & 0 & 0 \\
\hline Extraocular disease & 167 & 0 & 165 & 6 & 10 & 7 & 2 & 3 & 2 & 1 \\
\hline Total & 618 & & & 12 & 11 & 7 & & 10 & 2 & 1 \\
\hline
\end{tabular}

${ }^{a}$ Outcome in bilateral disease was dependent on the grouping of the worst eye. ${ }^{\mathrm{b}}$ One patient was group C to begin with but subsequently required enucleation in view of progression.

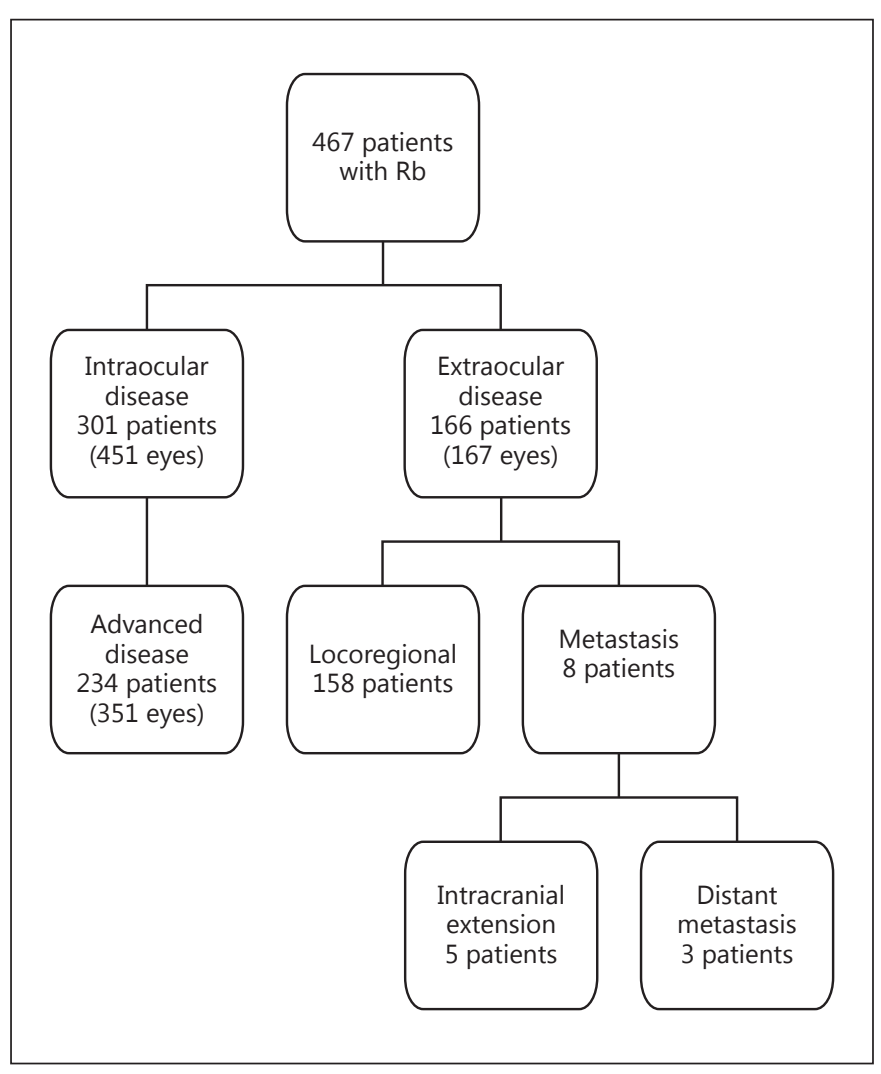

Fig. 1. Flowchart depicting the distribution and clinical grouping of patients. $\mathrm{Rb}$, retinoblastoma.

\section{Clinical Features}

The presenting features of retinoblastoma patients in the study cohort are summarized in Table 2 and the clinical grouping of patients is summarized in Table 3 and Fig- ure 1. Leukocoria and strabismus were the most common presenting features. There were 11 patients in group A; 46 patients in group B, 10 patients in group C, 28 patients in group D, and 206 patients in group $\mathrm{E}$ intraocular disease. Extraocular disease was present in 166 patients. It was interesting to note that $156(87.2 \%)$ females presented with advanced stage as compared to 233 males $(81.0 \%$; $p=$ 0.052 ). Patients who were below poverty line had more extraocular disease at presentation $(n=38 ; 40.8 \%)$ than those who were not $(n=86 ; 22.9 \%)(p=0.024)$.

\section{Treatment}

Three hundred and forty-seven patients (451 eyes; $74.3 \%$ ) accepted treatment, while 120 patients (25.6\%) refused any kind of treatment at presentation. The primary reason for refusal was the taboo of enucleation. Fifteen out of 347 had been treated elsewhere and were subsequently referred to our Institute. The initial and subsequent treatment received by these 347 patients is outlined in Figure 2. Three hundred and forty-nine eyes out of the 451 eyes needed enucleation, but only 278 underwent treatment. Enucleation acceptance rate was thus $79.7 \%$. Chemoreduction was given to 228 patients, while 23 received adjuvant chemotherapy. OPEC regimen was given to 134 , while 117 received VEC regimen. After evaluating the initial response to 2 or 3 cycles of chemotherapy, adjuvant local therapy was administered. All the patients in group D were treated by chemotherapy first, and globe salvage was possible in $17.0 \%$. For extraocular disease, secondary enucleation was done after 2-3 cycles of chemoreduction. The mean time from diagnosis to secondary enucleation was $2.3 \pm 0.4$ months (range: 6 weeks to 3 months). Seven patients received melphalan and 1 re- 
Fig. 2. A summary of the treatment received by the compliant patients. ${ }^{*}$ One bilateral patient with coexisting retinopathy of prematurity underwent primary laser coagulation. " Rest received it as an adjuvant therapy.

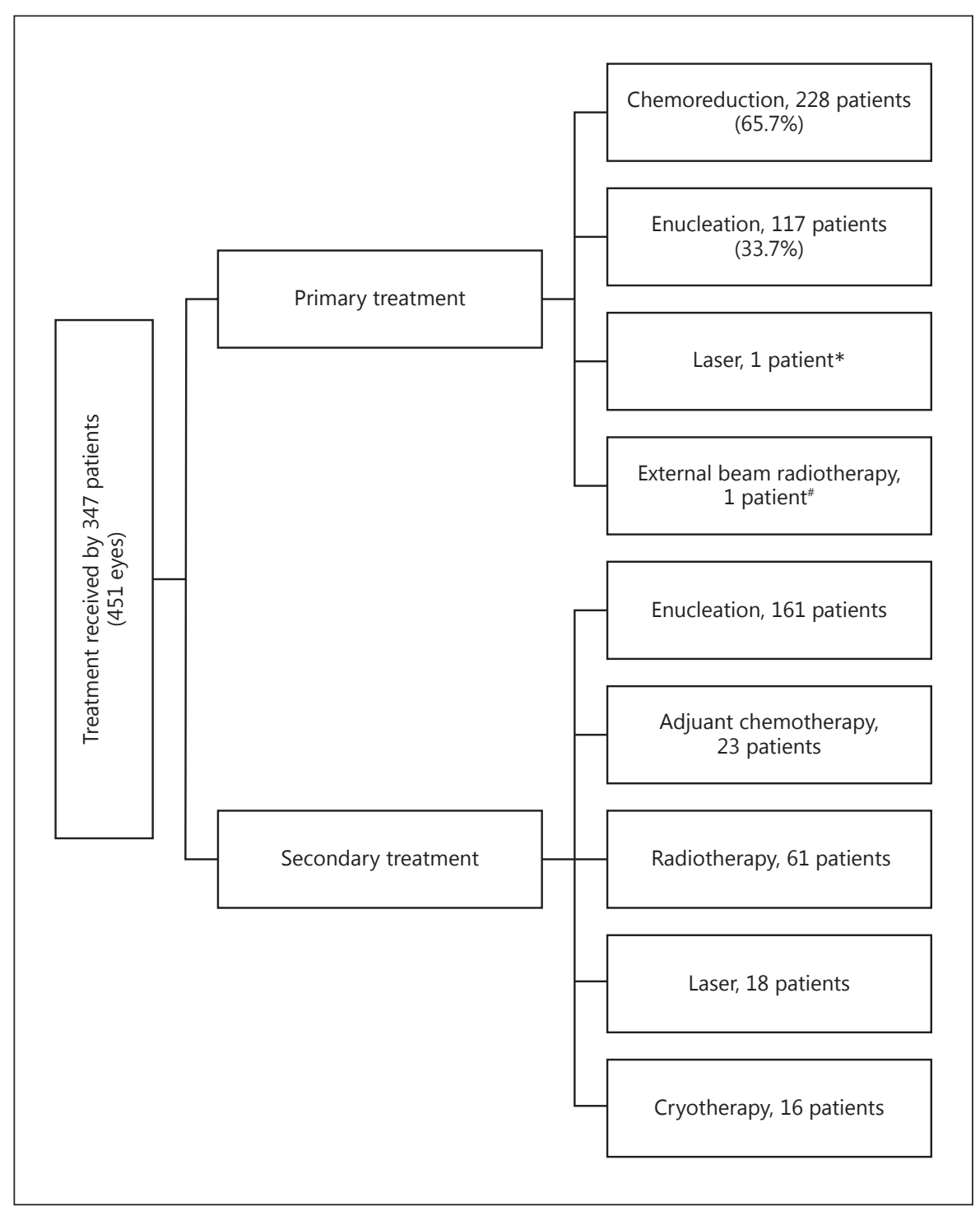

ceived subconjunctival carboplatin in addition to the primary treatment.

\section{Histopathology}

HP reports were retrievable for 245 eyes out of 278 enucleations (due to the study being retrospective in nature). At least one HRF was found in 31 eyes (17.8\%). Twelve eyes had more than one HRF. Retrolaminar optic nerve involvement was present in $22(70.9 \%)$, with a positive nerve resection limit in 8 (25.8\%); optic nerve with choroidal invasion was seen in 3 eyes $(9.7 \%)$, scleral extension in 4 (12.9\%), and anterior segment involvement with ciliary body invasion in 1 eye (3.2\%). None of the patients had $>3 \mathrm{~mm}$ choroidal invasion alone.

Sixteen-Year Review of Retinoblastoma
There were 177 eyes out of these 245 with available HP reports, who had intraocular retinoblastoma, and the rest had extraocular disease. Out of the 177 eyes, 77 eyes had undergone a primary enucleation. In this subset, 16 eyes (20.8\%) had HRF. Of the remaining 100 eyes that had undergone a secondary enucleation, $5(5.0 \%)$ had HRF. This difference of HRF in primary versus secondary enucleation in eyes with intraocular disease was found to be highly significant $(p<0.0004)$.

\section{Adverse Effects}

In the patients who received chemotherapy, a total of 17 adverse events were noted. These included anorexia, nausea, and vomiting in 3; anemia in 11; one each with

Ocul Oncol Pathol 2018;4:23-32 
Table 4. Subgroup analysis with major outcomes

\begin{tabular}{|c|c|c|c|c|c|}
\hline Subgroup & $n$ & Positive HRF & Globe salvage & Recurrences & Deaths \\
\hline \multicolumn{6}{|l|}{ Chemotherapy regimen } \\
\hline OPEC (before 2007) & 134 & $25(18.6)$ & $32(23.9)$ & $13(9.7)$ & $5(3.7)$ \\
\hline VEC (after 2007) & 117 & $7(5.9)$ & $36(30.8)$ & $2(1.7)$ & $6(5.1)$ \\
\hline$p$ value & & 0.061 & 0.42 & $0.001^{*}$ & 0.00 \\
\hline \multicolumn{6}{|l|}{$H R F$} \\
\hline Present & 31 & - & - & 5 & 13 \\
\hline Absent & 247 & - & - & 10 & 0 \\
\hline$p$ value & & & - & 0.352 & $0.009^{*}$ \\
\hline \multicolumn{6}{|c|}{ Poor socioeconomic status } \\
\hline Yes & 93 & $8(8.6)$ & $46(49.5)$ & $8(8.6)$ & $7(7.5)$ \\
\hline No & 374 & $23(6.2)$ & $228(60.9)$ & $5(1.3)$ & $6(1.6)$ \\
\hline$p$ value & & $0.0458^{*}$ & $0.041^{*}$ & $0.042^{*}$ & 0.054 \\
\hline \multicolumn{6}{|c|}{ Age $>5$ years at presentation } \\
\hline Yes & 37 & $19(51.3)$ & $13(35.1)$ & $16(43.2)$ & 7 (18.9) \\
\hline No & 437 & $12(2.7)$ & $187(42.8)$ & $4(0.9)$ & $6(1.37)$ \\
\hline$p$ value & & $0.032^{*}$ & 0.072 & $0.022^{*}$ & $0.023^{*}$ \\
\hline
\end{tabular}

Data are presented as $n(\%)$. HRF, high risk features. * Significant values.

seizures, neutropenia, and high-grade fever with oral ulcers. Out of these, 6 children (2.4\%) developed side effects requiring hospital admission (anemia requiring blood transfusion [3], seizures [1], and high-grade fever [1]).

\section{Outcome}

The mean follow-up was $28.5 \pm 44.4$ months (median $=64$ months; range 2-190 months). One hundred and fifty-one patients (43.5\%) defaulted during the course of therapy. Out of these, 21 (6.1\%) presented again with more advanced disease, whereas 130 (37.4\%) were subsequently lost to follow-up. Of those who completed therapy, 151 children (32.2\%) were tumor free at 5-year followup. Of the remaining children, 163 (34.9\%) were tumor free at the last visit but had still not completed their 5-year follow-up at the end of the study. Some functional vision was retained in $50(48.1 \%)$ eyes of bilateral retinoblastoma patients. Twenty-two (6.4\%) had local recurrence of the tumor. Two patients $(0.6 \%)$ had bony metastasis to the hip and arm, and 1 developed CNS metastasis at an average follow-up of 28.5 months. Thirteen (3.8\%) patients died, of which 12 had extraocular disease at presentation. One patient had group E disease but refused primary enucleation and presented again with extraocular disease. The average interval from presentation to death was 4.7 months (range $0-34$ months). A bilateral retino- blastoma patient developed second intracranial tumor (fibroblastic meningioma of sphenoid bone) 14 years after radiotherapy. He remains disease free 5 years after surgical excision of the tumor.

The outcome in various subgroups of patients is given in Table 4, and the difference in outcomes of primary enucleation versus chemoreduction is given in Table 5 . Though chemoreduction led to a significant decrease in the proportion of HRF, there was no significant difference in the outcome of disease in terms of death, recurrence or metastasis whether the patients received primary enucleation or chemoreduction first. All the late presenters $(n=4)$ had advanced disease at presentation. Two had bilateral disease with advanced disease in 1 eye requiring enucleation, one of which developed orbital recurrence after enucleation and subsequently was lost to follow-up; the other one remained disease free at the last follow-up. Two out of the late presenters had unilateral disease treated by enucleation.

\section{Discussion}

Retinoblastoma is the most common primary intraocular malignancy of childhood. The incidence of retinoblastoma varies across different regions of the world and represents almost $4 \%$ of all pediatric malignancies $[1,10]$. 
Table 5. Table showing differences in patients who received primary enucleation and systemic chemoreduction and subsequent development of metastasis and death

\begin{tabular}{lcll}
\hline & $\begin{array}{l}\text { Primary } \\
\text { enucleation }\end{array}$ & $\begin{array}{l}\text { Systemic } \\
\text { chemo- } \\
\text { reduction }\end{array}$ & $\begin{array}{l}p \\
\text { value }\end{array}$ \\
\hline $\begin{array}{l}\text { Total patients }(n=347) \\
\text { Unilateral/bilateral }\end{array}$ & 117 & 228 & \\
$\quad$ patients & $99 / 18$ & $144 / 84$ & \\
Group D, E & $102(87.1)$ & $99(43.4)$ & \\
Extraocular disease & $15(12.8)$ & $129(56.6)$ & $0.001^{*}$ \\
HRF intraocular Rb & $16(20.8)$ & $5(5)$ & $0.004^{*}$ \\
HRF extraocular Rb & $31(33.7)$ & $22(14.37)$ & $0.003^{*}$ \\
Metastasis & $1(0.9)$ & $7(3.1)$ & 0.559 \\
Recurrences & $10(8.8)$ & $12(5.26)$ & 0.415 \\
Death & $1(0.9)$ & $12(5.26)$ & 0.239 \\
\hline
\end{tabular}

Figures in parentheses indicate percentages. HRF, high-risk features; $\mathrm{Rb}$, retinoblastoma.

Approximately 250-300 children are newly diagnosed with retinoblastoma each year in the United States, and substantially higher rates occur in developing countries $[1,10]$. Retinoblastoma accounts for $2.5-4 \%$ of all childhood cancers in most developed countries, and a 2 - to 3 -fold higher incidence of tumors of the eye (majority of which are retinoblastoma in children $<15$ years of age) has been reported in India $[1,11,12]$. India still accounts for nearly one-third of retinoblastoma cases in the AsiaPacific region [13]. There is paucity of literature on epidemiological and clinical profile studies from India, with such large numbers. The present study reports a large number of retinoblastoma patients analyzed for sociodemographics, symptomatology, treatment, and outcome from India.

Our study reports a high number of new cases/year from a tertiary center with a large catchment area. The average age at presentation of our patients ( 35 months) is comparable to the range of $29-33$ months in previous reports from India $[3,12,14]$. It is also similar to studies from other medium Human Development Index (HDI) countries like Egypt [15] (36 months) and Mali [16] (50 months). HDI refers to a statistical measure of life expectancy, education, and income per capita used to rank the countries into 4 tiers of human development namely very high/high/medium/poor. In countries with very high $\mathrm{HDI}$, the average age at presentation is earlier with increasing numbers of affected children less than 1 year of age $[17,18]$. The advanced age at presentation in our

Sixteen-Year Review of Retinoblastoma study can be attributed to the fact that nearly $12.9 \%$ of the patients belonged to the lower socioeconomic strata and more than $50 \%$ were from a rural population. In our study, we found the delay in presentation to be up to 84 months. Since India is also a medium HDI country; lack of education/awareness may have resulted in the time lag between the onset of symptoms and presentation to our center [19]. Most often, the parents bring the child to the hospital only when they notice a white reflex (leukocoria) or when the child is old enough to complain about decreased visual acuity. The age difference between the unilateral and bilateral groups of patients in our study is also similar in most of the studies from developing countries. This difference is not only because of the fact that heritable retinoblastoma is usually bilateral with a lower age of presentation but also because of the above-mentioned medico-social factors [19].

Our study shows a male predominance (62\%), which is similar to the gender distribution shown in reports from Mexico [10] (52.4\%), Mali [16] (54.5\%), Egypt [17] (60.25), and Jordan [18] (70.0\%). There is a definite regional bias in studies from our country as males were generally preferred for treatment in earlier studies as well [3]. Being a tertiary care center, we largely get referrals of children from local hospitals, and it is alarming to note that health problems in female children are often ignored and undetected [20,21].

Family history of retinoblastoma was present in 19 patients $(4.09 \%)$, and this figure is comparable to $6.67 \%$ in Singapore [22] and 4.8\% in Iran [23]. Eleven percent of patients were aged more than 5 years at presentation, which is higher than 3.5\% reported from Brazil [17]. Gunalp et al. [24] in their study on a Turkish population reported that diagnosis at older age is a common finding in developing countries.

The same factors can be associated with a high incidence of local orbital spread and metastasis at presentation in our study (30.2\%), comparable to other studies from our country [3] and a figure much higher than in Western countries [25], where this figure drops to $10 \%$. None of our patients presented with retinoblastoma IIRC group A-C alone. Those with IIRC group D and $\mathrm{E}$ were incidentally detected with a lower group in the other eye. Advanced disease at presentation was seen in $77.8 \%$ of our patients. These children, along with those presenting with extraocular (27.0\%) disease at presentation, require complex and multimodal treatment. Radiotherapy and chemotherapy to salvage the globe in advanced cases predispose these children to poor survival and to secondary cancers [26]. A high proportion of ad-

Ocul Oncol Pathol 2018;4:23-32 DOI: $10.1159 / 000477408$ 
vanced disease at presentation was seen in previous studies from India $[3,27]$ as well as the rest of the developing world $[28,29]$. However, these figures are in contrast to the Western world $[26,30,31]$, where the incidence of extraocular disease has been reported to be less than $5 \%$.

In this study, only $17.8 \%$ had HRF in eyes that were affected by intraocular disease. There was a statistically significant difference in the HRF in primary versus secondary enucleation in intraocular retinoblastoma. The chemoreduction protocol could have been responsible for pathological downstaging of tumor as suggested by Zhao et al. [32]. Overall, HRF in postchemotherapy-reduced eyes were found in $10.1 \%$ (27/228) in our study, which is much lower than $51.9 \%$ reported by Suryawanshi et al. [33]. This gross difference is due to the inclusion of vitreous seeds in $44.2 \%$ as HRF and consideration of even extraocular cases for the study of HRF. In another study by Brennan et al. [34], the HRF were comparable in eyes undergoing primary and secondary enucleation, and the authors advocated prompt enucleation in advanced disease. Although the hypothesis that chemoreduction helps in downgrading the tumor grade is supported by our study figures, Zhao et al. [32] believe that this downstaging of pathological features by chemotherapy is associated with risk for extraocular extension being missed and less vigilant follow-up subsequently leading to high rates of metastasis and death. In a clinical scenario like ours, most of the patients presented with an advanced disease. Primary enucleation would be extremely difficult in an advanced disease and surgical maneuvers may lead to dissemination of the disease. Chemoreduction of the tumor makes surgical removal easier and feasible. It is important to note that we enucleate after giving 2-3 cycles of chemotherapy and do not wait until the completion of 6 cycles in patients who have advanced disease as in the above-mentioned study [32]. The authors also showed high risk of metastasis and orbital recurrences in case the systemic chemotherapy exceeded 3 months versus primary enucleation. In our study, the time period from the presentation to enucleation did not exceed 3 months in any patient undergoing secondary enucleation. We also give a chance of globe salvage to patients of bilateral retinoblastoma by chemoreduction, as evidenced by a $17.0 \%$ globe salvage rate in group $\mathrm{D}$ patients who would have otherwise required enucleation in our cohort. There was no significant difference in outcome in terms of recurrence, metastasis, and death if patients received chemoreduction first. The relatively higher but nonsignificant rates of death and metastasis in the group receiving chemoreduction are largely due to the fact that these patients had advanced disease to begin with, which per se increases the risk of metastasis and death. The results of our study are in accordance with the results of Berry et al. [35] that globe salvage therapy with systemic chemoreduction and subsequent enucleation for poor response does not increase the risk of metastatic disease or orbital recurrence. Larger future prospective trials will shed more light on this issue before guidelines are laid down.

Enucleation acceptance rate in our study was $79.6 \%$. In developing countries, enucleation refusal and default rate ranges from $13.7 \%$ in Taiwan [36], 22.0\% in Central America [37], 31.0\% in Malaysia [38], 33.0\% in Honduras [39] to $42.0 \%$ in Nigeria [40]. Treatment course was often punctuated by dropout and therapy defaulters. High proportions of extraocular disease $(27.0 \%)$, treatment dropouts (43.5\%), therapy defaulters (6.1\%), and refusal of initial treatment $(25.6 \%)$ in our study adversely affect patients' survival.

A significant proportion of children in our population need to be identified at an early stage of the disease and then treated with a timely and complete therapy. In a disease where no definitive genetic heterogeneity has been shown to correlate with outcomes [41], identification and early treatment form the mainstay for prognosis. Our study results suggest that in a clinical scenario like ours, where most of the disease is advanced at presentation, globe salvage attempt by chemoreduction does not increase the risk of recurrence, metastasis, and death.

Low awareness in rural areas, lack of understanding of the disease, extended family pressure, social taboo of enucleation, seeking alternative (herbal) conservative treatment options, and poor accessibility to health care play a critical role in delayed diagnosis in our population. There are high proportions of extraocular disease, treatment defaulters, and dropouts combined with refusal of initial treatment in our study; typical reasons include low socioeconomic strata, unwillingness for enucleation, and low priority given to the health of female children. The need of the hour is education and awareness among the common masses to reduce dropout in therapy and abandonment. There should be a combined effort from the government, medical practitioners, and social workers, to make the families of retinoblastoma patients understand the need for timely initiation and completion of recommended therapy as well as long-term follow-up.
30

Ocul Oncol Pathol 2018;4:23-32 DOI: $10.1159 / 000477408$
Singh/Katoch/Kaur/Dogra/Bansal/Kapoor 


\section{Statement of Ethics}

Institutional ethics committee approval was obtained for the study before reviewing the charts and the study conformed to the Declaration of Helsinki. Informed consent was obtained from parents for treatment at the time of diagnosis.

\section{Disclosure Statement}

The authors declare that there are no financial interests/conflicts of interest.

\section{References}

1 Shields CL, Shields JA: Diagnosis and management of retinoblastoma. Cancer Control 2004; 11:317-327.

2 Canturk S, Qaddoumi I, Khetan V, Ma Z, Furmanchuk A, Antoneli CB, Sultan I, Kebudi R, Sharma T, Rodriguez-Galindo C, Abramson DH, Chantada GL: Survival of retinoblastoma in less-developed countries impact of socioeconomic and health-related indicators. $\mathrm{Br} \mathrm{J}$ Ophthalmol 2010;94:1432-1436.

3 Chawla B, Hasan F, Azad R, Seth R, Upadhyay AD, Pathy S, Pandey RM: Clinical presentation and survival of retinoblastoma in Indian children. Br J Ophthalmol 2016;100: 172-178.

4 Sitorus RS, Moll AC, Suhardjono S, et al: The effect of therapy refusal against medical advice in retinoblastoma patients in a setting where treatment delays are common. Ophthalmic Genet 2009;30:31-36.

5 Sachdeva S: Trend of reported cases of retinoblastoma under five hospital based cancer registries. Indian J Cancer 2010;47:473-474.

6 Linn Murphree A: Intraocular retinoblastoma: the case for a new group classification. Ophthalmol Clin North Am 2005; 18:41-53.

7 Chantada G, Doz F, Antoneli CB, Grundy R, Clare Stannard FF, Dunkel IJ, Grabowski E, Leal-Leal C, Rodríguez-Galindo C, Schvartzman E, Popovic MB, Kremens B, Meadows AT, Zucker JM: A proposal for an international retinoblastoma staging system. Pediatr Blood Cancer 2006;47:801-805.

8 Chantada G, Luna-Fineman S, Sitorus RS, Kruger M, Israels T, Leal-Leal C, Bakhshi S, Qaddoumi I, Abramson DH, Doz F; SIOPPODC Graduated-Intensity Retinoblastoma Guidelines Writing Committee: SIOP-PODC recommendations for graduated-intensity treatment of retinoblastoma in developing countries. Pediatr Blood Cancer 2013;60: 719-727.

9 Munier FL, Gaillard MC, Balmer A, Soliman S, Podilsky G, Moulin AP, Beck-Popovic M: Intravitreal chemotherapy for vitreous disease in retinoblastoma revisited: from prohibition to conditional indications. Br J Ophthalmol 2012;96:1078-1083.

10 Leal-Leal C, Flores-Rojo M, Medina-Sansón A, Cerecedo-Díaz F, Sánchez-Félix S, González-Ramella O, Pérez-Pérez F, Gómez-Martínez R, Quero-Hernández A, Altamirano-Alvarez E, Alejo-González F, Figueroa-Carbajal
J, Ellis-Irigoyen A, Tejocote-Romero I, Cervantes-Paz R, Pantoja-Guillén F, Vega-Vega L, Carrete-Ramírez F: A multicentre report from the Mexican Retinoblastoma Group. Br J Ophthalmol 2004;88:1074-1077.

11 Arora RS, Eden T, Kapoor G: Epidemiology of childhood cancer in India. Indian J Cancer 2009;46:264-273.

12 Kumar A, Moulik NR, Mishra RK, Kumar D: Causes, outcome and prevention of abandonment in retinoblastoma in India. Pediatr Blood Cancer 2013;60:771-775.

13 Usmanov RH, Kivelä T: Predicted trends in the incidence of retinoblastoma in the AsiaPacific region. Asia Pac J Ophthalmol (Phila) 2014;3:151-157; erratum in: Asia Pac J Ophthalmol (Phila) 2014;3:397.

14 Kaliki S, Srinivasan V, Gupta A, Mishra DK, Naik MN: Clinical features predictive of highrisk retinoblastoma in 403 Asian Indian patients: a case-control study. Ophthalmology 2015;122:1165-1172.

15 Hassan SA, Massoud AA, Hussein GH: Retinoblastoma in Upper Egypt (1981-1991). Saudi J Ophthalmol 1992;6:32-38.

16 Boubacar T, Fatou S, Fousseyni T, Mariam S, Fatoumata DT, Toumani S, Abdoul-Aziz D, Marouf KM: A 30-month prospective study on the treatment of retinoblastoma in the Gabriel Toure Teaching Hospital, Bamako, Mali. Br J Ophthalmol 2010;94:467-469.

17 Bonanomi MT, Almeida MT, Cristofani LM, Odone Filho V: Retinoblastoma: a three-yearstudy at a Brazilian medical school hospital. Clinics (Sao Paulo) 2009;64:427-434.

18 Jaradat I, Yousef YA, Mehyar M, Sultan I, Khurma S, Al-Rawashded K, Wilson M, Qaddoumi I, Salem A, Alnawaiseh I: Retinoblastoma in Jordan: an epidemiological study (2006-2010). Hematol Oncol Stem Cell Ther 2011;4:126-131.

19 Dimaras H, Kimani K, Dimba EA, Gronsdahl P, White A, Chan HS, Gallie BL: Retinoblastoma. Lancet 2012;379:1436-1446.

20 Saha A, Parmar V, Chawala D, Walia D: Gender bias in utilization of health services in Chandigarh. Indian J Pediatr 2009;76:858.

21 Khera R, Jain S, Lodha R, Ramakrishnan S: Gender bias in child care and child health: global patterns. Arch Dis Child 2014;99:369374.

22 Lim FP, Soh SY, Iyer JV, Tan AM, Swati H, Quah BL: Clinical profile, management, and outcome of retinoblastoma in Singapore. J Pediatr Ophthalmol Strabismus 2013;50:106112.

23 Naseripour M, Nazari H, Bakhtiari P, Modarres-Zadeh M, Vosough P, Ausari M: Retinoblastoma in Iran: outcomes in terms of patients' survival and globe survival. $\mathrm{Br} \mathrm{J}$ Ophthalmol 2009;93:28-32.

24 Gunalp I, Gunduz K, Arslan Y: Retinoblastoma in Turkey: diagnosis and clinical characteristics. Ophthalmic Genet 1996;17:21-27.

25 Wong JR, Tucker MA, Kleinerman RA, Devesa SS: Retinoblastoma incidence patterns in the US Surveillance, Epidemiology, and End Results program. JAMA Ophthalmol 2014; 132:478-483.

26 Jubran RF, Erdreich-Epstein A, Butturini A, Murphree AL, Villablanca JG: Approaches to treatment for extraocular retinoblastoma: Children's Hospital Los Angeles experience. J Pediatr Hematol Oncol 2004;26:31-34.

27 Bakhshi S, Gupta S, Gogia V, Ravindranath Y: Compliance in retinoblastoma. Indian J Pediatr 2010;77:535-540.

28 Chang CY, Chiou TJ, Hwang B, Bai LY, Hsu WM, Hsieh YL: Retinoblastoma in Taiwan: survival rate and prognostic factors. Jpn J Ophthalmol 2006;50:242-249.

29 Essuman V, Ntim-Amponsah CT, Akafo S, Renner L, Edusei L: Presentation of retinoblastoma at a paediatric eye clinic in Ghana. Ghana Med J 2010;44:10-15.

30 Karcioglu ZA, al-Mesfer SA, Abboud E, Jabak MH, Mullaney PB: Workup for metastatic retinoblastoma. A review of 261 patients. Ophthalmology 1997;104:307-312.

31 Truong B, Green AL, Friedrich P, Ribeiro KB, Rodriguez-Galindo C: Ethnic, racial, and socioeconomic disparities in retinoblastoma. JAMA Pediatr 2015;169:1096-1104.

32 Zhao J, Dimaras H, Massey C, Xu X, Huang D, Li B, Chan HS, Gallie BL: Pre-enucleation chemotherapy for eyes severely affected by retinoblastoma masks risk of tumor extension and increases death from metastasis. J Clin Oncol 2011;29:845-851.

33 Suryawanshi P, Ramadwar M, Dikshit R, Kane SV, Kurkure P, Banavali S, Viswanathan S: A study of pathologic risk factors in postchemoreduced, enucleated specimens of advanced retinoblastomas in a developing country. Arch Pathol Lab Med 2011;135:10171023. 
34 Brennan RC, Qaddoumi I, Billups CA, Free TL, Haik BG, Rodriguez-Galindo C, Wilson MW: Comparison of high-risk histopathological features in eyes with primary or secondary enucleation for retinoblastoma. Br J Ophthalmol 2015;99:1366-1371.

35 Berry JL, Kogachi K, Aziz HA, McGovern K, Zolfaghari E, Murphree AL, Jubran R, Kim JW: Risk of metastasis and orbital recurrence in advanced retinoblastoma eyes treated with systemic chemoreduction versus primary enucleation. Pediatr Blood Cancer 2017;64.

36 Chen YH, Lin HY, Hsu WM, Lee SM, Cheng CY: Retinoblastoma in Taiwan: incidence and survival characteristics from 1979 to 2003. Eye (Lond) 2010;24:318-322.
37 Luna-Fineman S, Barnoya M, Bonilla M, Fu L, Baez F, Rodríguez-Galindo C: Retinoblastoma in Central America: report from the Central American Association of Pediatric Hematology Oncology (AHOPCA). Pediatr Blood Cancer 2012;58:545-550.

38 Menon BS, Alagaratnam J, Juraida E, Mohamed M, Ibrahim H, Naing NN: Late presentation of retinoblastoma in Malaysia. Pediatr Blood Cancer 2009;52:215-217.

39 Leander C, Fu LC, Peña A, Howard SC, Rodriguez-Galindo C, Wilimas JA, Ribeiro RC, Haik B: Impact of an education program on late diagnosis of retinoblastoma in Honduras. Pediatr Blood Cancer 2007;49:817-819.
40 Kodilinye HC: Retinoblastoma in Nigeria: problem of treatment. Am J Ophthalmol 1967;63:469-448.

41 Zhang J, Benavente CA, McEvoy J, FloresOtero J, Ding L, Chen X, Ulyanov A, Wu G, Wilson M, Wang J, Brennan R, Rusch M, Manning AL, Ma J, Easton J, Shurtleff S, Mullighan C, Pounds S, Mukatira S, Gupta P, Neale G, Zhao D, Lu C, Fulton RS, Fulton LL, Hong X, Dooling DJ, Ochoa K, Naeve C, Dyson NJ, Mardis ER, Bahrami A, Ellison D, Wilson RK, Downing JR, Dyer MA: A novel retinoblastoma therapy from genomic and epigenetic analyses. Nature 2012;481:329334. 